\title{
Analysis of Residual Stress Gradient in MEMS Multi-layer Structure
}

\author{
Jin Qian ${ }^{1}$, Ya-Pu Zhao ${ }^{1 *}$, Ru-Zeng Zhu ${ }^{1}$, Tong-Xi Yu ${ }^{2}$
}

1. State Key Laboratory of Nonlinear Mechanics (LNM), Institute of Mechanics, Chinese Academy of Sciences, Beijing 100080, China

2. Department of Mechanical Engineering, Hong Kong University of Science and Technology, Clear Water Bay, Kowloon, Hong Kong SAR, China

\begin{abstract}
Residual stress and its gradient through the thickness are among the most important properties of as-deposited films. Recently, a new mechanism based on a revised Thomas-Fermi-Dirac (TFD) model was proposed for the origin of intrinsic stress in solid films, giving the first order approximation of the stress gradient. The electron density at the boundary of the atoms (EDBA) defined by TFD model is taken as a dominating parameter inducing the stress. This paper applies the TFD model to multi-layer case, which is a typical structure in MEMS devices. The theoretical calculations suggest possibilities to control and reduce the residual stress.
\end{abstract}

Keywords: MEMS, thin films, multi-layer, residual stress, gradient, electron density, TFD

\section{Introduction}

Microelectromechanical Systems (MEMS) are among the most significant technological advances of this decade. Thin films are good candidates for fabrication of MEMS devices compatible with microelectronics technology. However, the process generally produces as-deposited films with high residual stress and residual stress gradient through the thickness, which can be detrimental to devices' performance. In order to obtain reliable thin films, residual stress and its gradient distribution must be identified and controlled.

Generally, residual stress in thin films is measured using wafer curvature method, in which the Stoney formula [1] serves as a cornerstone. The relationship between residual stress $\sigma_{f}$ and wafer curvature $\kappa$ is

$$
\sigma_{f}=\kappa M_{s} \frac{h_{s}^{2}}{6 h_{f}},
$$

where $M_{s}$ and $h_{s}$ are the biaxial elastic modulus and the thickness of the substrate, and $h_{f}$ is the thickness of the film. The formula does not presume any distribution of the film stress through the thickness, conflicting with the curving deformation of released structures.

As pointed out by Ommen et al [2], there are two major causes for the occurrence of stress in thin films: intrinsic stress due to the volume change during the silicidation reaction, and thermal stress due to the temperature change after the reaction. In general, thermal stress $\sigma_{t h}$ is uniform through the thickness and it can be calculated from [3]

$$
\sigma_{t h}=M_{f} \int\left[\alpha_{f}(T)-\alpha_{s}(T)\right] \mathrm{d} T
$$

where $M_{f}$ is the biaxial elastic modulus of the

\footnotetext{
${ }^{*}$ To whom all correspondence should be addressed. E-mail: yzhao@lnm.imech.ac.cn (Ya-Pu Zhao).
} 
film, $\alpha_{f}$ and $\alpha_{s}$ are the thermal expansion coefficients of the film and the substrate respectively. Recently, a new mechanism based on a revised Thomas-Fermi-Dirac (TFD) model was proposed for the origin of intrinsic stress in thin solid films [4]. According to this model, intrinsic stress in films is induced by the difference between the EDBAs of the film and the substrate. The occurrence of intrinsic stress is a requirement of the continuity of the EDBA at the interface.

\section{EDBA difference model}

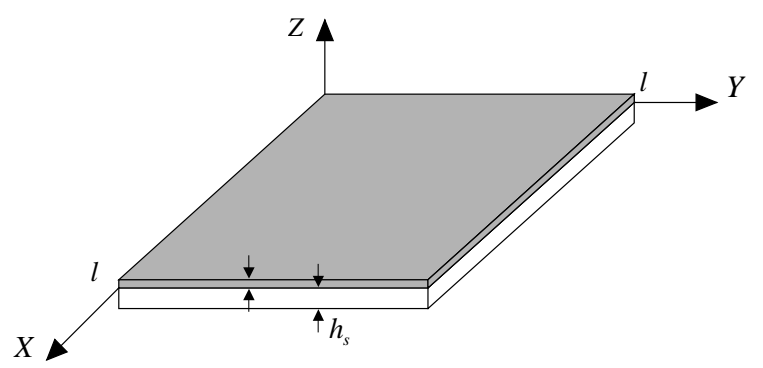

Fig. 1. Orientation of the rectangular coordinate with respect to a film of thickness $h_{f}$ and a substrate of thickness $h_{s}$.

A free film-substrate system is referred to a rectangular coordinate system oriented as shown in Fig. 1. The thickness of the film and the substrate are both much less than the lateral dimension $l$. Under the conditions stated, it follows that all shear components of stress and the normal stress in $Z$ direction can be neglected, and the normal components of stress in $X$ and $Y$ directions must be equal. Thus:

$$
\begin{gathered}
\sigma_{x x}=\sigma_{y y}=\sigma(z), \\
\sigma_{z z}=\tau_{x y}=\tau_{y z}=\tau_{x z}=0 .
\end{gathered}
$$

Eqs. (3a) and (3b) presume the bi-layer is invariant under translation in either $X$ direction or $Y$ direction.

The volume strain is

$$
\theta(z)=2 \sigma(z) /(3 \lambda+2 G)
$$

where $\lambda$ and $G$ are Lam é's constants, accords with the Laplacian equation:

$$
\Delta \theta(z)=0 \text {. }
$$

That is

$$
\frac{\partial^{2} \theta(z)}{\partial z^{2}}=0 .
$$

Therefore, $\theta(z)$ is linear through the thickness. Suppose

$$
\begin{aligned}
& \theta_{f}=\left(1-\frac{z}{h_{f}}\right) \theta_{f 0}, \\
& \theta_{s}=\left(1-\frac{z}{h_{s}}\right) \theta_{s 0},
\end{aligned}
$$

where the subscripts $f$ and $s$ denote the film and the substrate respectively, $\theta_{0}$ is the volume strain at the interface.

If the bi-layer is cut by a plane normal to the layer surface, then the absence of any externally applied loading implies that the net force must be zero, i.e.

$$
\int_{0}^{h_{f}} \sigma_{f}(z) \mathrm{d} z+\int_{0}^{h_{s}} \sigma_{s}(z) \mathrm{d} z=0 .
$$

Substituting the normal stress in term of the volume strain into Eq. (8) gives

$$
\frac{\theta_{f 0}}{\theta_{s 0}}=-\frac{\left(3 \lambda_{s}+2 G_{s}\right) h_{s}}{\left(3 \lambda_{f}+2 G_{f}\right) h_{f}} .
$$

At the interface $z=0$ and along either side, one has

$$
\begin{gathered}
\theta_{f 0}\left(3 \lambda_{f}+2 G_{f}\right)=3 p_{f}=3 \frac{\mathrm{d} p_{f}}{\mathrm{~d} n_{f}}\left(n-n_{f 0}\right), \\
\theta_{s 0}\left(3 \lambda_{s}+2 G_{s}\right)=3 p_{s}=3 \frac{\mathrm{d} p_{s}}{\mathrm{~d} n_{s}}\left(n-n_{s 0}\right),
\end{gathered}
$$

where $n$ is the EDBA at the interface, connected with pressure $p$ in the TFD model, and $p$ is the same as the pressure of the elastic equation. Combining Eqs. (9), (10a) and (10b), one gets the expression for $n$

$$
\frac{n-n_{f 0}}{n-n_{s 0}}=-\frac{h_{s} \mathrm{~d} p_{s} / \mathrm{d} n_{s}}{h_{f} \mathrm{~d} p_{f} / \mathrm{d} n_{f}}=-\frac{A_{s}}{A_{f}} .
$$

The stress in the film due to EDBA difference is

$$
\begin{aligned}
& \sigma_{f}=\frac{3}{2}\left(\frac{\mathrm{d} p}{\mathrm{~d} n}\right)_{f} \frac{A_{s}}{A_{s}+A_{f}}\left(n_{s 0}-n_{f 0}\right)\left(1-\frac{z}{h_{f}}\right) .(12) \\
& \text { If } h_{f}<<h_{s}, \text { it follows } A_{f}<<A_{s} \text {. The }
\end{aligned}
$$


intrinsic stress in the film is

$$
\sigma_{f}=\frac{3}{2}\left(\frac{\mathrm{d} p}{\mathrm{~d} n}\right)_{f}\left(n_{s 0}-n_{f 0}\right)\left(1-\frac{z}{h_{f}}\right) .
$$

The solution based on EDBA difference model gives the first order approximation of the stress gradient.

\section{EDBA difference model for multi-layer case}

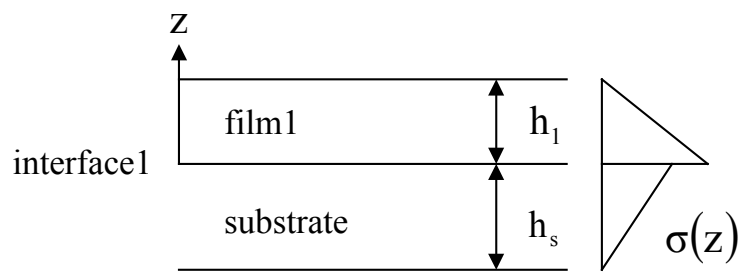

Fig. 2. The internal stress distribution due to the deposition of film1.

EDBA difference model gives the intrinsic stress distribution of the film-substrate bi-layer system. As an extension of the model, a general tri-layer system is considered. The internal stress distribution due to the first deposition, shown as Fig. 2, has been given above. Then attention will be focused on the additional internal stress due to the second deposition. Suppose

$$
\begin{aligned}
& \sigma_{2}(z)=\frac{(2 G+3 \lambda)_{2}}{2}\left(1-\frac{z}{h_{3}}\right) \theta_{20}, \\
& \left(0 \leq z \leq h_{2}\right) \\
& \sigma_{1}(z)=\frac{(2 G+3 \lambda)_{1}}{2}\left(1-\frac{z}{h_{1}+h_{s}}\right) \theta_{10}, \\
& \left(0 \leq z \leq h_{1}\right) \\
& \sigma_{s}(z)=\frac{(2 G+3 \lambda)_{s}}{2}\left(1-\frac{z}{h_{1}+h_{s}}\right) \theta_{10}, \\
& \left(h_{1} \leq z \leq h_{1}+h_{s}\right)
\end{aligned}
$$

where the subscripts 1,2 and $\mathrm{s}$ denote film 1 , film2 and substrate in Fig. 3 respectively, $\theta_{0}$ is the volume strain at interface2. Applying the condition imposed by overall equilibrium

$$
\int_{0}^{h_{2}} \sigma_{2}(z) \mathrm{d} z+\int_{0}^{h_{1}} \sigma_{1}(z) \mathrm{d} z+\int_{h_{1}}^{h_{1}+h_{s}} \sigma_{s}(z) \mathrm{d} z=0
$$

one has

$$
\frac{\theta_{20}}{\theta_{10}}=-\frac{\left[\begin{array}{l}
(2 G+3 \lambda)_{1} \frac{h_{1}^{2}+2 h_{1} h_{s}}{h_{1}+h_{s}}+ \\
(2 G+3 \lambda)_{s} \frac{h_{s}^{2}}{h_{1}+h_{s}}
\end{array}\right]}{(2 G+3 \lambda)_{2} h_{2}}=-\frac{A_{1, s}}{A_{2}} .
$$

At interface $2 z=0$ and along either side,

$$
\begin{aligned}
& \theta_{20}(3 \lambda+2 G)_{2}=3 p_{2}=3\left(\frac{\mathrm{d} p}{\mathrm{~d} n}\right)_{2}\left(n-n_{20}\right) \\
& \theta_{10}(3 \lambda+2 G)_{1}=3 p_{1}=3\left(\frac{\mathrm{d} p}{\mathrm{~d} n}\right)_{1}\left(n-n_{10}\right) .
\end{aligned}
$$

Substituting Eqs. (17a) and (17b) into Eq. (16) determines the EDBA at interface2, then the additional internal stress can be given by insertion the value of the EDBA into Eqs. (14a), (14b) and (14c). If $h_{1}, h_{2}<<h_{s}$, then the intrinsic stress in film2 is

$$
\sigma_{2}=\frac{3}{2}\left(\frac{\mathrm{d} p}{\mathrm{~d} n}\right)_{2}\left(n_{10}-n_{20}\right)\left(1-\frac{z}{h_{2}}\right) \text {. }
$$

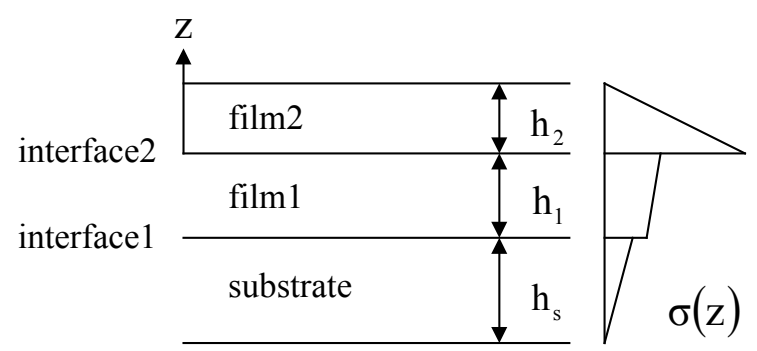

Fig. 3. The additional internal stress distribution due to the deposition of film2.

If film 1 is etched as a sacrificed layer, film2 will bend due to the internal stress gradient. The curvature-radius of the bending can be calculated as

$$
\rho=\frac{2}{3} M_{2} \frac{h_{2}}{(d p / d n)_{2}\left(n_{10}-n_{20}\right)},
$$

where $M_{2}$ is the biaxial elastic modulus of film2.

The total internal stress distribution of the tri-layer system should be the sum of the stress distributions in Fig. 2 and Fig. 3. Repeating similar course as above, results for structures 
containing more layers can be obtained. A computational procedure based on this model will be helpful because of the taxing calculation when the layer-number increasing.

\section{Discussion}

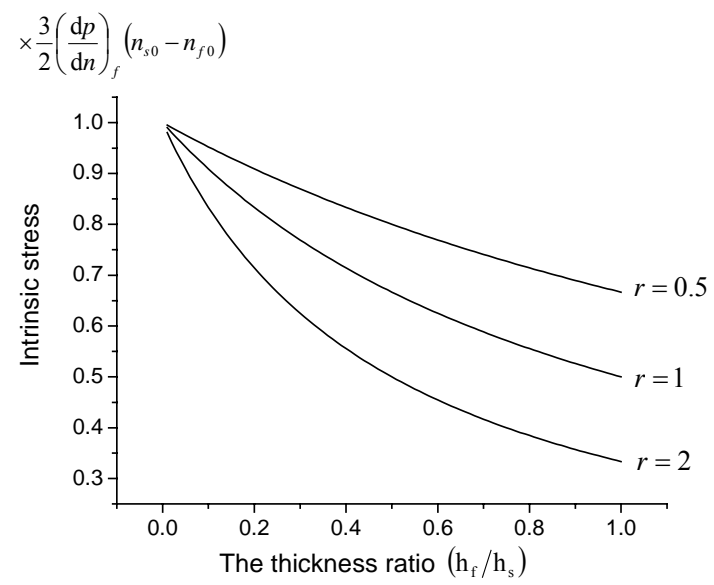

Fig. 4. The relationship between the thickness ratio $\left(h_{f} / h_{s}\right)$ and intrinsic stress. $\quad\left(r=\frac{(\mathrm{d} p / \mathrm{d} n)_{f}}{(\mathrm{~d} p / \mathrm{d} n)_{s}}\right)$

The expression of intrinsic stress in thin films, shown as Eq. (12), suggests possibilities to control and reduce it. In MEMS materials selection, films with relatively low value of $\mathrm{d} p / \mathrm{d} n$ are welcome preventing high intrinsic stress. In geometry design, because intrinsic stress is inverse proportional to the thickness ratio $h_{f} / h_{s}$, as shown in Fig. 4 , the reduction of film thickness will induce increase in stress. When the thickness ratio surpasses a critical value, the high stress will cause the film to tear off. In fabrication process, techniques reducing EDBA difference between the film and the substrate would be effective to diminish the intrinsic stress. Dose implantation has been taken to modify the EDBA of substrate by Liu et al [4], the experiment resulted in considerable reduction of intrinsic stress.

\section{Conclusions}

The following conclusions can be obtained:
- In thin films, the normal stress is much smaller than the lateral stresses. The lateral stresses have a linear distribution through the thickness.

- The thickness ratio $h_{f} / h_{s}$ is an important parameter in film design. When the ratio is lower than a critical value, stress will be high enough to tear film off.

- The magnitude of internal stress is proportional to the difference in electron density of the materials at interface and to the derivative of pressure with respect to electron density. Hence the stability of the composite demands a small difference in electron densities between the two layers at interface.

\section{Acknowledgments}

This research is supported by the National “973" Project, the National Natural Science Foundation of China and Key Project from CAS (Grant No. KJCX2-SW-L2).

\section{References}

[1] Stoney, G.G. The tension of metallic films deposited by electrolysis, Proc. Roy. Soc. London, 9(1909)172-175

[2] van Ommen, A.H., Bulle-Lieuwma, C.W.T., and Langereis, C, J. Appl. Phys, 64(1988)2706

[3] Hass, G., and Thun, T.E. Physics of thin films, Academic Press, New York, 1966

[4] Cheng, K.J., and Cheng, S.Y. Analysis and computation of the internal stress in thin films, Progress in Natural Science, 8(6)(1998)679-689

[5] Liu, J.F., Feng, J.Y., and Zhu, J. Micromechanism of intrinsic stress in $\mathrm{C}_{\mathrm{O}} \mathrm{Si}_{2}$ thin films, Progress in Natural Science, 11(2)(2001)163-167 (In Chinese) 